\title{
Transient paralysis after robotic prostatectomy
}

\author{
Samuel Deem $\cdot$ Cordell R. Davis $\cdot$ James P. Tierney
}

Received: 29 October 2007 / Accepted: 5 December 2007 / Published online: 9 January 2008

(C) Springer London 2008

\begin{abstract}
Laparoscopic radical prostatectomy (LARP) has been accepted as first line therapy for clinically localized prostate cancer. Complications have been low and outcomes are comparable to that of open surgery with potential benefits including shorter hospital stay, less pain and quicker return to normal activity. Unexplained paralysis following LARP is a rare entity with no reported cases in the current literature. We report a case of complete motor paralysis following LARP. An extensive multidisciplinary evaluation did not definitively establish a diagnosis. Aggressive multimodality treatment led to a complete recovery. Our understanding of this phenomena with the possible etiology and treatment is discussed.
\end{abstract}

Keywords Paralysis $\cdot$ Prostatectomy $\cdot$ Robotic · Complications

\section{Case presentation}

We report on a 63-year-old, average frame, Caucasian male found to have clinical stage T1c adenocarcinoma of the prostate who, after discussion of all options, elected to undergo laparoscopic radical prostatectomy (LARP). A

S. Deem $(\bowtie) \cdot$ C. R. Davis · J. P. Tierney

Urological Surgery Residency Program,

Charleston Area Medical Center,

3110 MacCorkle Ave. S.E., Charleston,

WV 25304, USA

e-mail: samuel_deem@yahoo.com

C. R. Davis

e-mail: cdavis@wvsom.edu

J. P. Tierney

e-mail: drjptierney@yahoo.com routine preoperative course then followed, including a medical history and physical examination, normal chest X-ray, electrocardiogram, basic metabolic panel and complete blood count.

Our patient was positioned carefully in concert with the surgery and anesthesia team. The procedure was performed with an operative time of $276 \mathrm{~min}$ and was without incident. Blood loss was approximately $200 \mathrm{ml}$, and the patient was hemodynamically stable throughout the entire procedure. After reversal of anesthesia and extubation, he was reported to be able to move all extremities upon arrival to the Post Anesthesia Care Unit (PACU). A few moments later he was unable to demonstrate any movement of all four extremities. He was alert and oriented and did not require re-intubation. The anesthesia personnel were asked to first exclude drug reaction or persistent neuromuscular blockade from muscle relaxants; neurology and spinal surgery consultation followed within the hour, and his sensation and muscle tone was found to be intact. Deep tendon reflexes were grade II throughout all extremities

A non-contrast computed tomography (CT) scan of the brain was negative for acute hemorrhage. Magnetic resonance imaging (MRI) of the brain, thoracic and cervical spine proved to be negative for spinal cord compression or injury, and a magnetic resonance arteriogram (MRA) of the brain showed no aneurysm or other lesion. Neurology and spinal surgery neurological findings were consistent with pure motor quadriplegia with a differential diagnosis including an anterior spinal cord infarct or ischemia not evidenced by the MRI. Spinal cord injury protocol was initiated including bolus dose steroids followed by continuous steroid infusion [1].

At 12 and $24 \mathrm{~h}$ post-surgery the patient still demonstrated complete motor quadriplegia. With the diagnosis of spinal injury becoming less favored, a trial of hyperbaric 
oxygen therapy to treat a presumptive diagnosis of gas air embolism (GAE) was requested. The first hyperbaric oxygen therapy was started approximately $26 \mathrm{~h}$ postoperatively and led to an immediate ability to move his upper extremities and some minimal movement of his lower extremities. Twelve hours later the second hyperbaric oxygen treatment appeared to further increase his motor function. An aggressive physical therapy regimen followed and over the course of the next 14 days a slow but steady recovery was appreciated. $\mathrm{He}$ is now more than 8 weeks postoperative and is fully recovered and functional in the community.

\section{Discussion}

Laparoscopic radical prostatectomy is rapidly becoming the procedure of choice for surgical treatment of clinically localized prostate cancer. Current recommended guidelines for positioning were followed and include having the patient in steep Trendelenberg position, the legs placed in stirrups, and the knees flexed and lowered. All pressure points are padded with foam pads to prevent injury and the neck remains aligned and supported in a neutral position [2]. An optimal position was achieved and maintained throughout the entire procedure. The prostatectomy was felt to be routine, and an excellent outcome was expected. Postoperatively our patient reported a remote history of minor hyperextension cervical spine injury that required no surgery and left him with no deficits. There was no evidence of congenital abnormality or injury on any of our imaging studies.

Reversible motor paralysis is not a known complication of LARP or other laparoscopic surgery that could be found in the current literature. However, there have been reports of specific deficits including paralysis in the literature that were attributed to GAE by other authors [3-5]. The lack of significant response to steroids in our case led us to doubt spinal cord injury as the explanation for these deficits. Negative MRI findings agreed with this conclusion, and a repeat MRI was performed 1 week postoperatively that showed no delayed findings consistent with injury. Our first discussion of a diagnosis of GAE or anterior spinal cord ischemia was entertained early on postoperative day one. With no current diagnostic modality to confirm this suspicion, a trial of hyperbaric oxygen therapy was suggested and with excellent response.

Most of the literature on GAE and treatment modalities with hyperbaric oxygen therapy is based on decompression sickness and scuba diving injuries [6-8]. Cockett et al. [7] demonstrated venous outflow obstruction as the cause of paralysis following induced decompression sickness in dogs. The dogs were treated with Dextran 70 with various outcomes prior to euthanization and autopsy. There was significant white matter destruction showing 'extensive spongy degeneration' and 'focal zones of demyelination' with sparing of the gray matter. These findings were felt to be consistent with venous occlusion from gas embolism leading to the paralysis. GAE has been shown to produce similar spinal cord syndromes in other reports and typically has responded well to hyperbaric oxygen therapy.

The pneumoperitoneum used in LARP could have resulted in venous entry of gas embolus eventually traveling to the inferior vena cava (IVC). Upon removal of the steep Trendelenberg position, the GAE could travel to the more proximal vena cava and cause obstruction of the lumbar veins and subsequently venous congestion compromising the spinal cord. This might explain the delay between the end of the procedure and the onset of motor paralysis. Although the diagnosis of GAE with spinal cord involvement was suspected, there is no typical presentation or firm method of diagnosis available. With a significant index of suspicion for a GAE and a rapid recovery with hyperbaric oxygen therapy, it was essentially a diagnosis of exclusion.

The pathophysiology affecting our patient is unknown and not easily explained. Although no immediate response was recognized, steroid administration could have been responsible for the eventual recovery as well. Regardless of the accurate etiology of the paralysis, we want to stress here the importance of early (i.e. $<8 \mathrm{~h}$ ) multidisciplinary evaluation and treatment. Treatment including steroid administration to cover spinal cord injury and hyperbaric oxygen therapy if GAE is a consideration could be critical in this rare event.

\section{References}

1. Bracken MB, Shepard MJ, Collins WF et al. (1990) Naloxone in the treatment of acute spinal cord injury: Results of the second national acute spinal cord injury study. New Eng J Med 322(20):1405-1411

2. Carey RI, Leveillee RJ (2007) Patient positioning for robotic urologic procedures. In: Patel VR (eds) Robotic urologic surgery. Springer, London, pp 61-66

3. Murphy BP, Harford FJ, Cramer FS (1985) Cerebral air embolism resulting from invasive medical procedures. Treatment with hyperbaric oxygen. Ann Surg 201(2):242-245

4. Heckmann JG, Lang CJG, Kindler K et al (2000) Neurologic manifestations of cerebral air embolism as a complication of central venous catheterization. Crit Care Med 28(5):1621-1625

5. Stolzenburg JU, Rabenalt R, Do M et al (2006) Complications of Endoscopic extraperitoneal radical prostatectomy (EERPE): prevention and management. World J Urol 24:668-675

6. Neuman TS, Bove AA (1990) Combined arterial gas embolism and decompression sickness following no-stop dives. Undersea Biomed Res 17(5):429-436

7. Cockett AT, Pauley SM, Zehl DN et al. (1979) Pathophysiology of bends and decompression sickness. An overview with emphasis on treatment. Arch Surg 114(3):296-301

8. Barrett DW (1996) Spinal air embolism resulting from a party game. Neurology 47(1):298-299 